\title{
Memantine Augmentation in a Down's Syndrome Adolescent with Treatment-Resistant Obsessive-Compulsive Disorder
}

\author{
Scott R. Pekrul, MD, and Kate D. Fitzgerald, MD
}

\section{To The Editor:}

D OWN'S SYNDROME (DS) IS A CHROMOSOMAL DISORDER that is the most frequent cause of intellectual disability (mental retardation $[\mathrm{MR}]$ ). Psychiatric comorbidity is relatively common in the Down's population, and is estimated to range from $18 \%$ to $38 \%$ (Prasher and Day 1995; Capone et al. 2006). Although little research has been completed with obsessive-compulsive disorder (OCD) in DS populations, it is estimated to occur in from $0.8 \%$ to $4.5 \%$ of patients (Prasher and Day 1995; Capone et al. 2006). OCD is characterized by intrusive thoughts, images, or urges (obsessions) with purposeful mental or physical actions (compulsions) aimed at reducing anxiety caused by the obsession. OCD in DS patients is postulated to present more often with compulsive behaviors of ordering and tidiness, such as rearranging personal belongs or opening/closing doors, cabinets, blinds, and light switches (Prasher and Day 1995; Capone et al. 2006), driven by "just right" obsessions (Charlot et al. 2002).

First-line pharmacological treatment for OCD in nondevelopmentally disordered populations is a serotonin reuptake inhibitor (SSRI), which is effective in 40-60\% of cases (Pediatric OCD Treatment Study [POTS] Team 2004; Abudy et al. 2011). Various strategies for treatment-resistant OCD include switching to another SSRI, augmentation with antipsychotics, or the use of a serotonin norepinephrine agent (Abudy et al. 2011). More recently, there is emerging evidence that glutamatergic agents such as memantine are effective in treatment-resistant OCD in both adult and pediatric OCD patients (Pasquni and Bodni 2006; Aboujaoude, Barry, Gamel, 2009; Hezel et al. 2009; Stewart et al. 2010). Given that memantine has demonstrated effectiveness in treatment-resistant OCD in nondevelopmentally disabled patients, it may be helpful in DS populations with OCD. Here, we report on a case using memantine in an adolescent with DS and OCD.

\section{Case Report}

$\mathrm{N}$ was a 16-year-old Caucasian male with a history of DS and mild MR who presented to a university-based child/adolescent psychiatry clinic with his biological parents for evaluation of obsessive behaviors. $\mathrm{N}$ had had no prior psychiatric history until age 14 when he developed "just right" rituals. His symptoms were characterized by compulsively turning off lights; insisting that all doors needed to be shut; as well as aligning books, pictures, boxes, and sunglasses so they were "just so." He also displayed hoarding behaviors such as keeping water bottles, sunglasses, and boxes. If a family member closed a door, turned on a light, or misarranged his items, he became very upset and agitated. He would engage in these behaviors up to 8 hours per day, causing significant distress in the home.

$\mathrm{N}$ was evaluated by a community psychiatrist who diagnosed him with OCD and initiated sertraline. He quickly developed agitation, anxiety, and increased compulsive behaviors. The medication was discontinued and changed to risperidone. On risperidone (dose unknown), he showed no change in symptoms and developed weight gain and drooling. Risperidone was then changed to aripiprazole, which he was on for several months before being evaluated at the university-based child/adolescent clinic.

At the university clinic evaluation, his parents reported that aripiprazole decreased his symptoms "somewhat" but that nonetheless the symptoms continued to cause significant distress. Given his minimal response and a $9.1 \mathrm{~kg}$ weight gain, it was tapered off. Another trial of an SSRI was agreed upon, and fluoxetine was initially titrated to $20 \mathrm{mg}$. After 4 weeks, the patient's symptoms improved with regard to allowing lights to be left on at night, but the remaining "just right" symptoms continued. Over the course of 6 months, fluoxetine was titrated to $100 \mathrm{mg}$, which led to a modest decrease in symptoms. He was continued on fluoxetine, and an incentive-based behavioral plan was initiated. This combination decreased his symptoms by $\sim 50 \%$. However, he continued to have prominent symptoms of opening/shutting windows and doors, turning lights on/off, and ordering furniture. Riluzole was added as an augmentation agent to fluoxetine. It was titrated to $50 \mathrm{mg}$ b.i.d., but his symptoms worsened and it was subsequently discontinued. His fluoxetine was crossed tapered with clomipramine and titrated up to $100 \mathrm{mg}$ over the course of $\sim 2$ months. It was determined, however, that his symptoms responded better to fluoxetine, and he was cross-tapered back to this medication. After fluoxetine was titrated to $80 \mathrm{mg}$ his OCD symptoms continued as before.

Memantine was added to augment fluoxetine. It was titrated to $15 \mathrm{mg}$ and the patient's fluoxetine was continued at $80 \mathrm{mg}$. Over the course of 4 months, N's symptoms significantly decreased. His compulsive behaviors of shutting doors/windows, turning on/off lights, and arranging items were reported to be "rare" by his parents. However, he continued with some hoarding behaviors. His progress was maintained over the course of 1 year.

\section{Discussion}

This case highlights the use of memantine as an augmenting agent in a DS patient with treatment-resistant OCD. N showed

Department of Child and Adolescent Psychiatry, University of Michigan Ann Arbor, Michigan. 
minimal response to and/or significant side effects from first-line SSRI agents as well as second-line strategies using atypical antipsychotics and a serotonin-norepinephrenine agent. An incentivebased behavioral protocol modified for N's developmental level combined with a high dose of fluoxetine demonstrated modest gains. However, it was not until N's treatment was augmented with memantine that any substantial improvement arose.

N's case highlights several important issues in treating DS patients with OCD. Diagnosing OCD in DS populations with developmental delay is challenging, as they often do not meet Diagnostic and Statistical Manual of Mental Disorders, 4th ed. (DSM-IV) criteria, and their developmental delay often hinders the use of diagnostic measures used for nondevelopmentally delayed patients (American Psychiatric Association 1994). Currently, there are no standard measures used to diagnosis or monitor symptoms in developmentally delayed patients with OCD. Diagnosing $\mathrm{N}$ was based on his symptoms of compulsive behaviors of ordering and symmetry that seemed to be driven by "just right" compulsions. This case, therefore, supports previous literature speculating that OCD in DS populations often presents with rearranging personal belongs or opening/closing doors, cabinets, blinds, and light switches (Prasher and Day 1995; Capone et al. 2006). Further research in the diagnosis of OCD in developmentally delayed patients is warranted.

There are few studies investigating the treatment of OCD in adult or pediatric DS patients. However, case reports have shown that SSRIs can be effective in this population (O'Dwyer et al. 1992; Sutor et al. 2006). Strategies for treating treatment-resistant patients with OCD and DS have emerged from treatment protocols in nondevelopmentally disordered populations, such as augmenting with atypical antipsychotics. Sutor et al. reported four cases of OCD in DS patients that responded to either an SSRI alone or a combination of an SSRI and risperidone (Sutor et al. 2006). However, beyond this, we found no further studies investigating treatment-resistant OCD in adult or pediatric DS populations.

Memantine is a glutamate receptor antagonist developed to slow the development of Alzheimer's disease (AD). In $\mathrm{AD}$, memantine is thought to decrease glutamate release by binding with a low affinity to the $N$-methyl-D-aspartate (NMDA) type receptor, which can ultimately inhibit the neurotoxic effects of prolonged calcium influx (Choi 1992; Parsons et al. 2007). Emerging lines of evidence have also implicated the role of glutamate dysfunction in the pathogenesis of OCD (Wu et al. 2012). Interestingly, patients with $\mathrm{DS}$ have an early onset of $\mathrm{AD}$, and memantine has been successfully used in these patients to slow cognitive decline (Prasher 2004; Mohan, Bennett, Carpenter, 2009). Several case reports (Poyurovsky et al. 2005; Hezel et al. 2006; Pasquini and Biondi 2006; Aboujaoude, Barry, Gamel, 2009) as well as a recent single-blinded case-control study (Stewart et al. 2010) have further demonstrated memantine as an effective augmenting agent in treatment-resistant OCD patients.

The glutamenergic theory for the pathogenesis of OCD supports the use of memantine as a novel agent (Wu et al. 2012; Pittenger, Bloch, Williams, 2011). It is notable that memantine has been used to slow cognitive decline in DS populations with early-onset $\mathrm{AD}$, presumably by decreasing the neurotoxic effects of increased glutamate release, as has been stated (Prasher 2004). Whether there is a direct correlation between $\mathrm{AD}$ and $\mathrm{OCD}$ through a common dysfunction in the glutamate pathway remains to be seen. Although memantine has been used to treat cognitive decline in $\mathrm{DS}$ populations with $\mathrm{AD}$, this is the first report that we are aware of for its use for DS patients with OCD.
Another glutamate agent, riluzole, is also used in DS populations with $\mathrm{AD}$ as well as being an emerging agent in treatment-resistant OCD (Prahser 2004; Coric et al. 2005; Grant et al. 2007). In this case, riluzole caused side effects, rendering it ineffective for our patient. Why $\mathrm{N}$ was not able to tolerate one glutamate agent and showed a robust response to the other is unknown. However, memantine and riluzole are believed to have different mechanisms of actions that may explain the differing effects. This case, therefore, suggests considering another glutamate agent if the first one is not tolerated.

\section{Disclosures}

No competing financial interests exist.

\section{References}

Aboujaoude E, Barry JJ, Gamel N: Memantine augmentation in treatment resistant obsessive-compulsive disorder: An open-label trial. J Clin Psychopharmacol 29:51-55, 2009.

Abudy A, Juven-Wetzler A, Zohar J. Pharmacological management of treatment-resistant obsessive-compulsive disorder. CNS Drugs 25:585596, 2011.

American Psychiatric Association: Diagnostic and Statistical Manual of Mental Disorders, 4th ed. Washington, DC: American Psychiatric Association; 1994.

Capone G, Goyal P, Ares W, Lannigan E: Neurobehavioral disorders in children, adolescents, and young adults with Down syndrome. Am J Med Genet C Semin Med Genet142C:158-172, 2006.

Charlot L, Fox S, Friedlander R: Obsessional slowness in Down's syndrome. J Intellect Disabil Res 46:517-524, 2002.

Choi DW. Excitotoxic cell death. J Neurobiol 23:1261-1276, 1992.

Coric V, Taskiran S, Pittenger C, Wasylink S, Mathalon DH, Valentine G, Saksa J, Wu YT, Gueorguieva R, Sanacora G, Malison RT, Krystal JH: Riluzole augmentation in treatment-resistant obsessive-compulsive disorder: An open-label trial. Biol Psychiatry 58:424-428, 2005.

Grant P, Lougee L, Hirschtritt M, Swedo SE. An open-label trial of riluzole, a glutamate antagonist, in children with treatment-resistant obsessive-compulsive disorder. J Child Adolesc Psychopharmacol 17:761-767, 2007.

Hezel DM, Beattie K, Stewart SE. Memantine as an augmenting agent for severe pediatric OCD. Am J Psychiatry 166:237, 2009.

Mohan M, Bennett C, Carpenter PK: Memantine for dementia in people with Down syndrom. Cochrane Database of Stematic Reviews 2009, Issue 1, Art. No: CD07657. DOI: 10.1002/14651858 .CD007657.

O'Dwyer J, Holmes J, Collacott RA. Two cases of obsessive-compulsive disorder in individuals with Down's syndrome. J Nerv Ment Dis 180:603-604, 1992.

Parsons CG, Stöffler A, Danysz W: Memantine: A NMDA receptor antagonist that improves memory by restoration of homeostasis in the glutamatergic system - too little activation is bad, too much is even worse. Neuropharmacology 53:699-723, 2007.

Pasquini M, Biondi M. Memantine augmentation for refractory obsessivecompulsive disorder. Prog Neuropsychopharmacol Biol Psychiatry 30: 1173-1175, 2006.

Pediatric OCD Treatment Study (POTS) Team: Cognitive-behavior therapy, sertraline, and their combination for children and adolescents with obsessive-compulsive disorder: The Pediatric OCD Treatment Study (POTS) randomized controlled trial. JAMA292:1969-1976, 2004.

Pittenger C, Bloch MH, Williams K. Glutamate abnormalities in obsessive compulsive disorder: Neurobiology, pathophysiology, and treatment. Pharmacol Ther 132:314-332, 2011. 
Poyurovsky M, Weizman R, Weizman A, Koran L: Memantine for treatment-resistant OCD. Am J Psychiatry 162:2191-2192, 2005.

Prasher VP: Review of donepezil, rivastigmine, galantamine and memantine for the treatment of dementia in Alzheimer's disease in adults with Down syndrome: Implications for the intellectual disability population. Int J Geriatr Psychiatry 19:509-515, 2004.

Prasher VP, Day S: Brief report: obsessive-compulsive disorder in adults with Down's Syndrome. J Autism Dev Disord 25:453-458, 1995.

Stewart SE, Jenike EA, Hezel DM, Stack DE, Dodman NH, Shuster L, Jenike MA: A single-blinded case-control study of memantine in severe obsessive-compulsive disorder. J Clin Psychopharmacol 30:34-39, 2010.

Sutor B, Hansen MR, Black JL. Obsessive compulsive disorder treatment in patients with Down syndrome: a case series. Downs Syndr Res Pract 10:1-3, 2006.
Wu K, Hanna GL, Rosenberg DR, Arnold PD. The role of glutamate signaling in the pathogenesis and treatment of obsessivecompulsive disorder. Pharmacol Biochem Behav 100:726-735, 2012 .

Address correspondence to: Scott R. Pekrul, MD Department of Psychiatry University of Michigan 4250 Plymouth Rd. SPC 5766 Ann Arbor, MI 48109

E-mail: scottpek@med.umich.edu 
This article has been cited by:

1. Ali Sahraian, Leila Razeghian Jahromi, Ahmad Ghanizadeh, Arash Mowla. 2017. Memantine as an Adjuvant Treatment for Obsessive Compulsive Symptoms in Manic Phase of Bipolar Disorder. Journal of Clinical Psychopharmacology 37:2, 246-249. [CrossRef] 